\title{
UNA PROPUESTA MODAL SOBRE LA T-TEORICIDAD Y EL PAPEL DE LOS POSTULADOS Y AXIOMAS EN LAS TEORÍAS CIENTÍFICAS
}

\author{
MANUEL DAHLQUIST \\ Universidad Nacional del Litoral \\ Universidad Autónoma entre Ríos \\ manuel.dahlquist@gmail.com \\ ADRIANA GONZALO \\ Universidad Nacional del Litoral \\ CONICET \\ adriana.n.gonzalo@gmail.com
}

RESUMEN: El denominado “problema de los términos teóricos" que inicialmente formuló Sneed (1971) surge de la interpretación que de los términos teóricos hace la concepción heredada. Esta lectura no se condice con la interpretación indirecta de los términos teóricos que Carnap realiza $(1937,1939)$ al utilizar postulados. Estos textos proponen una interpretación holística de las teorías científicas, sólo adecuadamente tratada en una semántica de mundos posibles. Andreas $(2008,2010)$ proporciona un tratamiento modal de los términos teóricos; nosotros presentamos estos resultados en términos de modelos de Kripke; luego los extendemos con el fin de que sean capaces de distinguir entre axiomas y oraciones teóricas, generando un modelo capaz de tratarlos de manera diferenciada y que aporte nuevos resultados lógicos y filosóficos. PALABRAS CLAVE: Carnap, concepción heredada, interpretación indirecta, lógica modal, términos teóricos

SUMM ARY: The so-called "problem of theoretical terms" first formulated by Sneed (1971) arises from the interpretation of theoretical terms according to the Inherited Conception. This reading is not consistent with the indirect interpretation of theoretical terms made by Carnap $(1937,1939)$ using postulates. These texts propose a holistic interpretation of scientific theories, that can only be properly treated in possible-world semantics. Andreas $(2008,2010)$ provides a modal treatment of theoretical terms; we present these results in terms of Kripkean models; then extend them so as to distinguish between axioms and theoretical sentences, generating a model capable of treating them in a differentiated matter and offering new logical and philosophical results.

KEY WORDS: Carnap, received view, interpretation indirect, modal logic, theoretical terms

\section{Introducción}

Este trabajo tiene su origen en una revisión de la interpretación que del problema de los términos teóricos hace la denominada concepción heredada (CH) de las teorías científicas (véase la sección 2.1.). Como es sabido, esta denominación se adopta convencionalmente a partir 
de Putnam (1962) para designar la concepción de la ciencia propia del empirismo lógico. Las reconstrucciones históricas de la CH son diversas, pero ha primado una "versión oficial" de la misma, que se popularizó a partir de Suppe (1974). Las ideas que fundan esa versión oficializada de la CH se basan particularmente en los trabajos de Carl Hempel y de una lectura parcializada de la obra de Rudolf Carnap.

La revisión que se mencionó anteriormente requiere algunas precisiones terminológicas en relación con la expresión "teórico". A la misma se le puede interpretar en al menos dos sentidos dentro del ámbito de la filosofía de la ciencia. En la llamada versión oficial se presenta una caracterización de la noción de término teórico, en contraste con la de término observacional (con la consabida diferenciación entre entidades observacionales y entidades teóricas). Así, un término es observacional si denota o designa entidades o propiedades del mundo fenoménico que pueden ser conocidas por observación directa; los términos teóricos, en contraste con aquellos, se conciben simplemente como el grupo de expresiones que se aplican (hacen referencia) a entidades o propiedades no observables. Algunos ejemplos paradigmáticos de dichas entidades son: electrones, neutrinos, fuerzas gravitatorias, genes, etc.

Existe además otra noción de teoricidad: un término teórico es aquel cuyo significado está determinado a través de los axiomas de una teoría científica. El significado del término "fuerza", por ejemplo, lo determinan las leyes de Newton para el movimiento y otras leyes acerca de las fuerzas especiales, tales como la ley de la gravitación. La siguiente definición de Andreas caracteriza este último sentido de teoricidad:

Un término t es teórico con respecto a una teoría $\mathrm{T}$, o T-teórico, sii no existe una aplicación de $\mathrm{T}$ en la cual el valor de verdad de t pueda ser determinado independientemente de los axiomas de T. Una aplicación de $\mathrm{T}$ es una aplicación de ciertos axiomas de $\mathrm{T}$ a un sistema de entidades empíricas. $\left(2013\right.$, p. 7) ${ }^{1}$

Esta última interpretación de la noción de "teórico" —en la que nos concentraremos a lo largo de este trabajo - resulta clave al momento de comprender el problema que Sneed (1971) ha señalado, y que se conoce como el "problema de los términos teóricos". En la sección 2.2. comentaremos dicho problema.

La revisión de la temática en la que nos enfocamos la examinaron muchos autores, entre ellos Friedman (1999, 2009), Coffa (1991) y

${ }^{1}$ La traducción de todas las citas de este artículo es nuestra. 
Andreas $(2007,2008,2010)$, quienes han señalado que, cuando analizamos las obras tempranas de Carnap $\left(1934 / 1937,{ }^{2} 1939\right)$ encontramos una caracterización de la interpretación de los términos teóricos — de aquí en más $t t$ - que claramente se distancia de la versión oficial. En la sección 3 analizaremos los textos carnapianos ya mencionados, para efectos de demostrar las interpretaciones de Friedman y Andreas que aquí seguimos. Sostenemos que efectuar una "lectura revisada" de estos textos, nos permite, por un lado, entender de manera diferente el problema de la interpretación de los $t$, lo que habilita una nueva lectura del legado del vienés, abandonando el esquema simplificador de lectura habitual de la CH y, por el otro, articular dicha lectura con propuestas actuales.

Andreas desarrolla en los textos antes mencionados una solución al denominado "problema de los términos teóricos" en términos de la semántica modal. La originalidad de la propuesta se basa, en gran parte, en mostrar cómo las deficiencias de una caracterización de los $t t$ en el marco de la semántica de primer orden pueden superarse en el marco de una semántica modal. En la sección 4 exponemos y defendemos la factibilidad de la perspectiva de Andreas, y mostramos las ventajas de un enfoque modal para el desarrollo de una semántica apropiada para enunciados que contienen $t t$.

Después, en la sección 5, señalamos las ventajas de la posición de Andreas, así como algunas limitaciones de la misma y damos una solución que amplía el modelo del mencionado autor (sección 6). Nuestra propuesta permite distinguir - en el modelo - los axiomas de las oraciones teóricas, algo que no se puede lograr en la propuesta original de Andreas. Asimismo, avanzamos desde la semántica de mundos posibles a la incorporación de elementos sintácticos que mejoran la representación de lo pretendido en estas nuevas interpretaciones de la $\mathrm{CH}$.

Finalmente, la sección 7 presenta un conjunto de conclusiones, con el que se intenta señalar que: a) el denominado "problema de los términos teóricos" en la CH, que inicialmente formula Sneed (1971), se apoya en la "visión oficial" de la misma; pero no se condice con la interpretación de los términos teóricos (términos-t) que aparece en Carnap (1937, 1939); b) que estas ideas se condicen con una interpretación holística de las teorías científicas, que sólo

${ }^{2}$ La obra de Carnap originalmente se publicó en alemán en 1934 bajo el título de Logische Syntax der Sprache, y posteriormente se editó en inglés en 1937 como The Logical Syntax of Language. La versión que utilizaremos es esta última, Carnap 1937. 
puede ser bien estudiada semánticamente en el marco de la semántica de mundos posibles; c) que este marco teórico puede generalizarse para avanzar sobre el tratamiento modal de problemas vinculados con los componentes pragmáticos necesarios para la buena representación de un teoría científica.

\section{La concepción heredada y el problema de los términos teóricos}

\subsection{La concepción de los $t t$ en la visión oficial de la CH}

Siguiendo la conocida versión de Frederick Suppe (1974) de la concepción heredada (CH) de las teorías científicas, ésta puede caracterizarse como sigue: ${ }^{3}$

La $\mathrm{CH}$ ha concebido las teorías como sistemas de enunciados expresables en términos de un lenguaje de primer orden L, y un cálculo lógico $\mathrm{K}$ asociado a $\mathrm{L}$.

El Lenguaje L de la teoría se divide en dos clases: i) un vocabulario formal; ii) un vocabulario no formal, en el que se distingue: (ii $\left.{ }_{a}\right)$ un vocabulario teórico $V_{t}$, que contiene sólo términos teóricos, (iii $)$ un vocabulario observacional $V_{o}$, que contiene sólo los términos de observación.

Asociado al lenguaje observacional $L_{o}$, existe un cálculo asociado $K_{o}$, que es la restricción de $K$ a $L_{o}$. $K_{o}$ debe admitir al menos un modelo finito y debe ser tal que todo término no- $V_{o}$ de $L_{o}$ esté explícitamente definido en $K_{o}$. Asimismo, existe un lenguaje de observación ampliado lógicamente, $L_{0^{\prime}}$, formado a partir de $L_{o}$, al que se han añadido los cuantificadores, operadores, etc., de $L$. Su cálculo asociado $K_{o^{\prime}}$ es la restricción de $K$ a $L_{o^{\prime}}$. Por otro lado, el lenguaje teórico, $L_{t}$, es el sublenguaje de $L$ que no contiene términos $V_{o}$; su cálculo asociado $K_{t}$, es la restricción de $K$ a $L_{t}{ }^{4}$ Por otra parte, en cada uno de los sublenguajes encontramos un conjunto de predicados y/o de variables funcionales que coincide en $L_{o}$ y $L_{o^{\prime}}$, pero que difiere en $L_{t}$.

Como condiciones para interpretar $L_{o}$ se requiere que: (a) el dominio de interpretación de $L_{o}$ y el cálculo $K_{o}$ asociado conste de acontecimientos, entidades y momentos concretos y observables; así como de propiedades y relaciones que hagan referencia a situaciones

${ }^{3}$ El texto no es una cita textual de Suppe (1974, p. 71 y ss.).

${ }^{4}$ Como Suppe hace notar (1974, p. 72) los sublenguajes descritos separadamente no agotan L, porque L también contiene enunciados mixtos; aquellos en los que al menos aparece un término $V_{t}$ y otro $V_{o}$. 
directamente observables; (b) el valor de cada variable $L_{o}$ se designe mediante una expresión de $L_{0}$.

Por su parte, la interpretación parcial de los $t t$ y de los enunciados de $L$ que los contienen, se logra mediante dos clases de postulados: los postulados teóricos $\mathrm{T}$ (esto es, los axiomas de la teoría) en que sólo aparecen los términos de $V_{t}$, y las reglas de correspondencia o postulados C. Estas últimas son enunciados mixtos y deben satisfacer las siguientes condiciones: (a) el conjunto total de éstas debe ser finito; (b) $\mathrm{C}$ debe ser lógicamente compatible con $\mathrm{T}$; (c) $\mathrm{C}$ no contiene términos extralógicos que no pertenezcan a $V_{o}$ o $V_{t}$; (d) cada regla de $\mathrm{C}$ debe contener esencial, o no insustancialmente, al menos un término $V_{o}$ y al menos otro $V_{t}$.

Dado $\mathrm{T}$, como la suma de postulados teóricos y $\mathrm{C}$ la de las reglas de correspondencia, entonces la teoría científica, basada en $L, \mathrm{~T}$, y $\mathrm{C}$ consiste en la suma de $\mathrm{T}$ y $\mathrm{C}$, y es designada como "TC". 5

2.2. El carácter paradojal de la solución de la CH y el problema de los $t t$ según Sneed

Sneed (1979, p. 31 y ss.) hacía notar un grave problema inherente a la concepción propia de la CH. Este problema hace referencia a la mutua dependencia de la extensión de los axiomas de una teoría científica y la extensión de los términos teóricos. Sneed percibe el carácter problemático de la determinación del "significado" de un $t t$ en una solución lingüística que identifica básicamente la expresión con la de "extensión" del término (1971, pp. 36-38).

Andreas plantea el problema en estos términos:

i) Existe al menos un término $t$ tal que sus valores pueden ser determinados sii asumimos la verdad de las sentencias que son

${ }^{5}$ Cabe mencionar que la anterior es una versión "normalizada" de la CH. Dentro de ésta existen además otras versiones en relación con la temática. Por ejemplo, el trabajo de Hempel (1970) resulta novedoso en relación con la versión sintetizada. El autor introduce las expresiones "principios internos" y "principios puente". Con los primeros se hará uso de un vocabulario teórico $V_{t}$, que contiene términos que no se emplean en las descripciones anteriores, ni en las generalizaciones acerca de los fenómenos empíricos que $\mathrm{T}$ debe explicar, sino que se introducen específicamente para caracterizar el escenario teórico y sus leyes, a diferencia de los segundos que contendrán tanto los términos de $V_{t}$ como aquellos del vocabulario que se usó para formar las descripciones originales y las generalizaciones de los fenómenos que la teoría debe explicar. Así, Hempel habla de un vocabulario disponible antes de que se introduzca la teoría, y concibe que su uso estará gobernado por principios que, al menos inicialmente, son independientes de aquélla, y se refiere a este vocabulario como preteórico, o previo, relativo a la teoría en cuestión" (Hempel 1970, pp. 142143). 
axiomas de la teoría T. Sea $\Phi_{T C}$ el conjunto de estas sentencias; t es o bien un predicado, o bien un símbolo de función, mas no una constante;

ii) el valor de verdad del conjunto de sentencias de $\Phi_{T C}$ son determinables, sii el valor del término t ya está dado (2008, p. 316).

Así, por un lado, debemos conocer la extensión de un término teórico $t$ a fin de averiguar si los enunciados sentencias de $\Phi_{T C}$ son verdaderos. Por el otro, resulta imposible determinar la extensión de $t$ sin asumir la verdad de algún enunciado de $\Phi_{T C}$ de antemano.

Sneed (1971) denominó a este círculo epistemológico "el problema de términos teóricos". Esta denominación ya no se usa aquí, a diferencia del uso de la expresión en la CH, para plantear el problema de la determinación del significado de los términos $t t$, sino para señalar la problematicidad de la solución linguística de la CH a la cuestión, o mejor dicho el carácter paradójico de la misma. ${ }^{6}$

\section{El problema de los términos teóricos y la solución de Carnap 1937-1939}

3.1. Sintaxis lógica, semántica y teoría de la ciencia

En Logical Syntax of Language (LSL) y - fundamentalmente- en Foundations of Mathematical and Logic (FML), Carnap presenta un enfoque del problema de los $t t$ que, como mostraremos, se aleja de la lectura que al comienzo del artículo mencionamos como interpretación oficial de la CH.

Por cuestiones de espacio trabajaremos básicamente con FML, aunque en LSL hay importantes novedades que afectan la interpretación de los $t t .{ }^{7}$ En lo que sigue expondremos un conjunto de ideas ex-

${ }^{6}$ Cabe consignar que Sneed (1971, p. 37) alude que el problema de que la caracterización de los términos teóricos en la $\mathrm{CH}$ podría caer en un círculo vicioso o en un regreso al infinito, dependiendo del número, finito o infinito de las aplicaciones intencionales que tengan las leyes presentes en los modelos de una teoría en cuestión.

${ }^{7}$ Como muestra, encontramos en la sección 82 de Logical Syntax of Language (LSL) que Carnap prescinde del principio de verificabilidad completa que había sostenido previamente, y admite la tesis de que las leyes de la naturaleza son enunciados de las teorías científicas — abandonando así la idea de que son reglas de construcción de oraciones de esas mismas teorías, tal como lo había planteado en 1928- Asimismo, como señala Acero (1994), Carnap da un paso inequívoco en la liberalización de su concepción de un sistema constitucional de conceptos al incluir conceptos o símbolos primitivos, es decir, conceptos o símbolos que "no tienen por qué ser reducibles por medio de una cadena de definiciones a los símbolos que aparecen en oraciones protocolares". 
puestas en FLM, vitales para dilucidar la noción de interpretación indirecta, imprescindible para entender la novedad de la propuesta acerca de la interpretación de los $t t$ que Carnap formula en esta época (Friedman 2009, p. 249).

\subsection{Sistemas semánticos y referencia directa}

Según Carnap, dado un lenguaje objeto extensional Sl y un lenguaje intensional $\mathrm{S} 2$, existe un metalenguaje $\mathrm{M}$ en el cual se pueden traducir todas las expresiones de los dos primeros. Así, las oraciones verdaderas del lenguaje objeto son verdaderas sólo porque ya eran lógicamente verdaderas en el metalenguaje (Carnap 1939, sección 5, pp. 29-31). Esta "relatividad metalingüística" es una característica inherente al enfoque semántico carnapiano (Awodey 2007, p. 235 y ss.).

Dado un lenguaje B - un lenguaje desconocido que habla un grupo de personas - podemos entenderlo a partir de estudios pragmáticos. "El lenguaje B pertenece al mundo de los hechos; tiene muchas propiedades, algunas de las cuales hemos descubierto, mientras que otras nos son desconocidas" (Carnap 1939, pp. 5-7). Los sistemas semánticos serán el lugar apropiado para tratar la relación entre las expresiones de un lenguaje y sus designata. "En ellos" estudiamos las relaciones entre las expresiones de B y sus designata. Sobre la base de esos hechos vamos a dictar un conjunto de reglas que establezcan estas relaciones. Las llamamos reglas semánticas (1939, p. 6). Éstas se construyen para el sistema B-S (de expresiones y sus designata) con el fin de mostrar cómo deben formularse las reglas semánticas y su función para determinar condiciones de verdad y de ese modo dar una interpretación de las sentencias de un sistema. A diferencia de B, B-S es un lenguaje construido, sólo tiene las propiedades que queremos que tenga. Los lenguajes de este tipo contienen símbolos lógicos y descriptivos. Los signos descriptivos se dividen en nombres y predicados y éstas son las reglas que constituyen el sistema semántico de este lenguaje artificial.

Reglas B-SD Designata de los signos descriptivos:

S-D 1: Los nombres designan cosas

S-D 2: Los predicados designan propiedades de las cosas

Reglas B-SL Condiciones de verdad para las sentencias de B-S:

Las reglas B-SL involucran signos lógicos. Las denominadas reglas L-semánticas de B-S. 
SL 1. "ist", forma Fl. Un enunciado de la forma "... ist... ${ }_{p}$ " es verdadero si y sólo sí la cosa designada por "...n" tiene la propiedad designada por "...p". (Carnap 1939, p. 9)

La transcripción de las expresiones carnapianas hace posible una caracterización ajustada de lo que el autor denomina interpretación directa de los $t$. Efectivamente, a partir de algunas de las reglas del sistema semántico se pueden establecer las condiciones de verdad (interpretar un enunciado) de manera directa.

Carnap dice: "Las reglas SL1, en combinación con SD, proveen condiciones de verdad directas para los enunciados de forma simple; directas, puesto que la regla no se refiere a la verdad de otros enunciados" y agrega:

Si se da un enunciado de B-S, se puede fácilmente construir, con la ayuda de las reglas dadas un criterio de verdad directa, esto es, una condición suficiente y necesaria para su verdad, de tal modo que en la formulación de esta condición no se haga ninguna referencia a la verdad de otras oraciones. $(1939$, p. 10)

Ahora bien, si una oración de un lenguaje objeto $\mathrm{S}$ es verdadera, lo es en virtud de su verdad en el metalenguaje $\mathbf{M}$ - como señalamos anteriormente- ${ }^{8}$ Así, siguiendo a Andreas, podemos presentar la siguiente definición de interpretación directa:

Interpretación directa: la interpretación de un símbolo es directa sii está dada por una interpretación en el metalenguaje que le otorga una intensión o una extensión. (Andreas 2008, p. 320)

\subsection{Cálculo e interpretación}

En FLM, Carnap presenta por primera vez su noción de interpretación indirecta para los que denomina "términos abstractos", en contraste con aquellos que se relacionan con la experiencia de manera directa. Su propuesta se presenta en el marco de la caracterización del cálculo. Parte de caracterizar un sistema sintáctico o cálculo como

${ }^{8}$ El metalenguaje puede ser formal o informal; Carnap habitualmente opta por la segunda opción. En un ejemplo que Davidson da (1963, p. 315) una sentencia como "F.B $=\mathrm{H}$ " del lenguaje objeto afirma que toda y cada una de los bípedos implumes son seres humanos. La traducción para "F.B" en el metalenguaje M es "bípedo implume", la traducción para "H" es "Humano", "=" se traduce por "equivalente en M"; por lo tanto la sentencia, puede ser traducida en M como: "Bípedo implume es equivalente a humano". 
un sistema de reglas formales que determinan ciertas propiedades y relaciones formales de enunciados, especialmente para el propósito de la deducción formal. El procedimiento más simple para la construcción de un cálculo consiste en establecer algunos enunciados como primitivos (llamados algunas veces axiomas o postulados) y algunas reglas de inferencia (Carnap 1939, p. 22).

Carnap distingue entre los cálculos dos tipos diferentes: los cálculos lógicos y los cálculos no lógicos. La diferencia estriba en su interpretación: los cálculos lógicos son (en la terminología de Carnap) L-determinados, esto es, tienen una interpretación puramente lógica. Los cálculos no lógicos, no. "Todos los cálculos no lógicos que expondremos más adelante constan, estrictamente hablando, de dos partes: un cálculo básico lógico y un cálculo específico añadido a él." El cálculo lógico es similar para todos los cálculos, a continuación agrega: "Lo que usualmente se llama un sistema de axiomas es, por consiguiente, la segunda parte de un cálculo cuyo carácter de parte no se advierte" (1939, p. 38).

En el apartado "Los cálculos y su aplicación a las ciencias empíricas" de FML se distingue entre: (i) interpretación del cálculo matemático; (ii) interpretación de la geometría; y (iii) interpretación de la física. Para nuestros propósitos nos detendremos en (iii), pues allí aparecen los dos tipos de interpretaciones — directa e indirectay se propone la segunda para los $t$. El cálculo físico es un cálculo básico "al que se le añaden los signos primitivos específicos y los axiomas, es decir, los enunciados específicos primitivos del cálculo físico en cuestión".

La interpretación también puede darse de manera indirecta (II), i.e., mediante reglas semánticas, no para los signos primitivos, sino para ciertos signos definidos del cálculo (cfr. FML § 24). En una de las últimas consideraciones que Carnap formula en esta sección de FLM se presenta una diferencia entre términos abstractos y términos elementales. El autor sostiene:

Encontramos entre los conceptos de la ciencia física - e igualmente entre los de toda la ciencia empírica - diferencias en cuanto a la abstracción. Algunos son más elementales que otros en cuanto a que podemos aplicarlos en casos concretos sobre la base de observaciones de un modo más directo que otros. Hay otros que son más abstractos.

Según Carnap existen grados de abstracción, y aunque no los define aquí, sí da una lista de términos a manera de guía: luminoso, oscuro, 
frío, dulce, azul, refiriéndose a los elementales; longitud, masa, densidad de presión, frecuencia de oscilación, en cuanto a los abstractos (p. 61).

En este apartado Carnap se plantea la "dirección" en la que debe construirse un cálculo para la Física. Si se debe partir de términos elementales (términos de un grado de abstracción mínima) o si, por el contrario, se debe partir de términos abstractos y "descender" hasta los términos elementales. Concluye que los físicos trabajan de la segunda manera (p. 64). De ahí su propuesta de considerar reglas de interpretación para los términos abstractos, que no pueden ser las mismas que para los términos elementales:

Si por el contrario, se toman como primitivos términos abstractos — de acuerdo con el único método empleado en la física científica-, entonces las reglas semánticas no tienen relación directa con los términos primitivos del sistema, sino que se refieren a términos introducidos por largas cadenas de definiciones. Primero se construye el cálculo flotando en el aire, valga la expresión: la construcción comienza por la parte superior y después se añaden niveles cada vez más bajos. Finalmente, mediante las reglas semánticas. El nivel inferior queda anclado al terreno sólido de los hechos observables. Las leyes ya sean generales o especiales, no se interpretan directamente, sino únicamente los enunciados singulares. Para los términos más abstractos, las reglas sólo determinan una interpretación indirecta, que es incompleta en cierto sentido. (p. 65; las cursivas son del original) ${ }^{9}$

Podemos, entonces, dar una definición general de interpretación indirecta siguiendo nuevamente a Andreas:

Interpretación indirecta: La interpretación de un símbolo es indirecta sii está dada por oraciones del lenguaje objeto que tienen el estatus de axiomas no lógicos del cálculo (i.e., son postulados) (2008, p. 322).

\footnotetext{
${ }^{9}$ Friedman vincula el tipo de dependencia semántica de los términos abstractos con las definiciones implícitas; en FLM para Carnap "las teorías de la física matemática moderna operan con términos 'abstractos' que se definen implícitamente, a la manera de Hilbert, en un sistema axiomático (y por lo tanto no requieren ningún sentido 'intuitivo' o "visualizable'), pero que todavía se refieren a fenómenos empíri$\cos$ (mediciones experimentales) de manera indirecta" (Friedman 2011, p. 258).
} 


\section{De Carnap a los desarrollos modales para la caracterización de los términos teóricos}

\subsection{Definiciones, postulados e interpretación holística}

Siguiendo la presentación de los elementos que componen un cálculo lógico aplicado, Carnap introduce la distinción entre interpretación indirecta mediante postulados y mediante definiciones (1939, pp. 6166).

Las definiciones tienen la función de proporcionar una interpretación indirecta a símbolos no primitivos de un sistema. Los postulados funcionan de manera análoga para la interpretación de un término teórico, aunque - a diferencia de las definiciones - puede pasar que, para un sistema $\mathrm{T}$, los $t t$ sean:

i) acumulativos: si eliminamos un término teórico tendremos algún tipo de pérdida epistémica en el sistema;

ii) no eliminables: no tendremos la misma potencia expresiva si contamos con él en el lenguaje que si no lo hacemos.

Los postulados son axiomas no lógicos que son tomados como válidos en el cálculo, y pueden, por ende, ser usados en cualquier derivación. La distinción entre axiomas lógicos y no lógicos está bien asentada en la tradición:

Esta técnica de sumar una premisa cuya verdad se justifica por el método axiomático de significados de los predicados es uno de los aspectos de lo que se conoce como el método axiomático. A menudo es posible cerrar la brecha entre la noción intuitiva de consecuencia y la noción más restringida de consecuencia de primer orden, expresando sistemáticamente datos sobre los predicados involucrados en nuestras inferencias. Las frases que se utilizan para expresar estos hechos algunas veces son llamadas postulados de significado, ${ }^{10}$ un tipo especial de axioma. (Barwise y Etchemendy 1999, p. 284)

${ }^{10}$ Carnap mismo propone esta manera de designar los postulados. Lo hace en un texto donde atiende el problema de los enunciados analíticos y dice que quien construye un sistema con postulados es libre de proponer los que desee "guiado, no por sus creencias con respecto a los hechos del mundo, sino por sus intenciones con respecto a los significados, i.e., la manera de usar las constantes descriptivas (1952, p. 225). 
Así, los postulados pueden considerarse axiomas no lógicos que imponen constraints a las valuaciones admisibles de los símbolos no lógicos del lenguaje. Un procedimiento familiar desde la perspectiva axiomática. De acuerdo con Andreas (2008) podemos afirmar que lo anterior significa — en términos de una semántica modelo-teóricaque cualquier estructura admisible de $\mathrm{L}$ debe satisfacer a aquellas oraciones que tienen como estatus ser definidas en el sistema respectivo. Los valores de estos términos están, en nuestro caso, ceñidos a los valores de los términos observacionales que se asumen para ser determinados en la interpretación directa (p. 321).

Tenemos, pues, que existe una única determinación de la extensión de un término definido mediante definición, como también de los valores de los términos primitivos. Por contraste, en la interpretación indirecta de un término por postulados obtenemos sólo una constricción impuesta sobre los valores admisibles de los $t t$ que no implica una única determinación. Los significados de los $t t$ pueden estar conformados por varios postulados, lo que refleja el hecho de que hay usualmente varios métodos de uso para uno y el mismo concepto científico. La interpretación para los $t t$ podría considerarse, entonces, holística.

\subsection{Una interpretación modal de los $t t$}

Los textos de Andreas $(2007,2008,2010)$ se han encaminado a defender una relectura de la CH a partir de los textos de Carnap (1937, 1939), y desde allí muestran las posibilidades de conciliación entre los desarrollos de Carnap en dichos textos y una nueva solución al problema de la determinación de los tt. Específicamente, Andreas (2009) propone dar a la idea más bien sintáctica de Carnap de interpretación a través de postulados, un viraje semántico que se sirve de lo hecho en el campo de la lógica modal.

La propuesta muestra la viabilidad de una lectura de la noción de T-teoricidad en términos modales, conciliable con la posición de Carnap en las obras referidas. La solución posee -como la de Ramsey - el mérito de superar el problema de los $t$, pero agrega la ventaja de estar expresada en una semántica matemáticamente bien estudiada (como la semántica de mundos posibles) y es, por lo mismo, insertable en modelos modales, carentes de la complejidad de la lógica de orden superior y aptos para probar la eficacia inferencial del modelo. La presentaremos formalmente más adelante. Antes demos cuenta de las ideas que fundan la solución. 
A diferencia de Andreas, nosotros nos expresaremos utilizando la notación canónica de estas semánticas, tanto para hacer la presentación del autor como para formular un análisis crítico y determinar la extensión del modelo; esto permite un acceso más rápido al trabajo y deja más claro su vínculo con el resto de la literatura modal.

Las nociones básicas para entender el resto de la propuesta lógica son: estructura relacional, marco, modelo, el lenguaje modal básico y su semántica. Recurriremos a lo que ya se puede considerar un texto clásico que revela los nuevos rumbos de los enfoques modales. La notación de la que se hace uso es la más difundida en la actualidad en la literatura modal. ${ }^{11}$

Definición 1: Una estructura relacional es un tuplo cuyo primer componente es un conjunto no vacío denominado el universo (o dominio), y cuyos demás componentes son relaciones sobre ese conjunto. Asumimos que toda estructura relacional contiene al menos una relación (Blackburn et al. 2001, p. 2).

Definición 2: Al lenguaje modal básico se le define utilizando un conjunto $\Phi$ de letras proposicionales cuyos elementos son usualmente denotados por $p, q, r$, más un operador unario $\square$ "caja". ${ }^{12}$ Las fórmulas bien formadas $\phi$ del lenguaje son dadas por la regla

$$
\varphi::=p|\perp| \neg \varphi|\psi \vee \varphi| \square \varphi
$$

Donde el rango de $p$ se establece sobre los elementos de $\Phi$ (Blackburn et al. 2001, p. 9).

Definición 3: Un marco para un lenguaje modal básico es un par $\mathcal{F}=(\mathrm{W}, \mathrm{R})$ donde

i) W es un conjunto no vacío

ii) $\mathrm{R}$ es una relación binaria sobre $\mathrm{W}$ (Blackburn et al. 2001, p. 18).

Definición 4: Un modelo para el lenguaje modal básico es un par $\mathcal{M}=(\mathcal{F}, \mathrm{V})$, donde $\mathcal{F}$ es un marco $\mathcal{F}=(\mathbb{W}, \mathrm{R})$ y $\mathrm{V}$ es una función

${ }^{11}$ Blackburn y Venema 2001.

${ }^{12}$ En el original el operador modal tomado como primitivo es el diamante; nosotros, en pro del argumento, proponemos como primitivo el operador caja. Esto no reviste mayor importancia ya que los operadores son duales, esto es, pueden definirse uno en virtud del otro sirviéndose de la negación, de la siguiente manera: $\diamond \varphi:=\neg[] \neg \varphi$. 
asignando a cada letra proposicional $p$ un subconjunto $\mathrm{V}(p)$ de $\mathrm{W}$. Informalmente pensamos el conjunto de puntos en nuestro modelo como siendo verdaderos (Blackburn et al. 2001, p. 17).

Definición 5: Asumiendo que $w$ es un estado en un modelo $\mathcal{M}=$ (W, R, V), entonces definimos inductivamente la noción de fórmula satisfecha (o verdadera) en un estado $w$ como:

(i) $\mathcal{M}, w \models p \quad$ sii $w \in \mathrm{V}(p)$ donde $p \in \varphi$

(ii) $\mathcal{M}, w \models \perp \quad$ sii nunca es el caso

(iii) $\mathcal{M}, w \models \varphi \wedge \psi \quad$ sii $\mathcal{M}, w \models \varphi$ y $\mathcal{M}, w \models \psi$

(iv) $\mathcal{M}, w \models \neg \varphi \quad$ sii no se da que $\mathcal{M}, w \models \varphi$

(v) $\mathcal{M}, w \models \square \varphi \quad$ sii para todo $\nu \in \mathbb{W}$ tal que $\nu \operatorname{R} w$ en $\mathcal{M}, \nu \models \varphi$

El giro semántico que Andreas propone se basa en que, si, como dijimos con anterioridad, los $t t$ son términos que imponen constraints sobre las interpretaciones, esto es, son términos verdaderos en un conjunto de interpretaciones escogidas, entonces su comportamiento semántico no difiere del que prescribe el operador modal " $\square$ " expresado en la cláusula (v) de la definición 5.

Volvamos ahora a la función de los postulados en la presentación de Andreas. Aceptamos — siguiendo a Carnap y a Ramsey - un lenguaje con dos niveles. Así, Andreas presenta la siguiente división de los postulados:

(i) Postulados-C que contienen términos observacionales así como términos teóricos.

(ii) Postulados- $\mathrm{T}$ que contienen solamente $t t$ como símbolos no lógicos.

Al conjunto de postulados-T también se le conoce como la teoría pura, mientras que a la suma de los conjuntos de postulados $\mathrm{C}$ y $\mathrm{T}$ se le denomina la teoría interpretada. Entre los T-postulados se encuentran las aserciones generales que se consideran las leyes de la naturaleza en un sentido no técnico (Andreas 2008, p. 319). 
El conjunto de oraciones que contienen los postulados T así como los C son representadas como $\Phi_{T C}$. La función básica de los $T C$ postulados es restringir el conjunto de interpretaciones admisibles a aquellas donde los TC-postulados son verdaderos.

La divergencia filosófica más interesante de esta perspectiva con la semántica estándar (como adelantamos en la sección 3.4.) consiste en rechazar la hipótesis de una única interpretación prevista de una teoría científica. Una vez que estamos convencidos de que hay $t$, es decir, símbolos no lógicos que obtienen su significado determinado a través de los postulados de una teoría científica, el requerimiento de una única interpretación —esencial a la semántica estándar- ya no se sostiene más.

Los elementos de los que formalmente se sirve pueden presentarse así:

Sea $u$ una estructura y sea $\mathrm{S}$ el conjunto de todas las estructuras $u \in \mathrm{S}$ que comparten las restricciones propuestas a la interpretación (esto es, el conjunto de interpretaciones admisibles o que hacen verdaderos a los $\Phi_{T C}$ postulados). Dado un lenguaje $L\left(V_{o}, V_{t}\right)$ como el descrito en la sección 2.1., entonces hay una función $\nu(\varphi)$ tal que:

Definición 6: $\nu(\varphi)$ asigna valores de verdad a los enunciados teóricos $L\left(V_{o}, V_{t}\right)$ y se define como sigue:

(i) $\nu(\varphi):=T$ sii para toda estructura $u \in \mathrm{S}, u \models \varphi$

(ii) $\nu(\varphi):=F$ sii para toda estructura $u \in \mathrm{S}, u \models \varphi$ no es válido

(iii) $\nu(\varphi)$ es indeterminada sii existe al menos una estructura $u_{1}$, para la cual es verdadero

$u_{1} \models \varphi$ y al menos otra estructura $u_{2}$ en la cual $u_{2} \models \varphi$ no sea verdadero, donde $u_{1}$ y $u_{2}$ son miembros de $\mathrm{S}$, el conjunto de estructuras admisibles (Andreas 2010, p. 373).

Como puede verse, ser verdadero en el conjunto de interpretaciones S, coincide con la idea básica de la lógica modal donde $\square \varphi$ es verdadera sii es verdadera en todos los (estados) mundos posibles accesibles desde (relacionados con) ese mundo. Sucede que "en lógica modal la interpretación de una fórmula no es un valor de verdad, sino el conjunto formado por todos los estados o mundos donde la fórmula es verdadera" (Manzano 2006, p. 206), como dejamos claro en la primera cláusula de la definición 5. En el caso que nos ocupa, la interpretación de una oración teórica es el conjunto de estructuras interpretativas $u \in S$ en que esas oraciones teóricas son verdaderas. 
Reconsiderando la Definición 6 en términos de la semántica de mundos posibles diríamos que: dado un modelo $\mathcal{M}=\langle\mathrm{S}, \mathrm{R}, \mathrm{V}\rangle$, donde $\mathrm{S}$ es un conjunto no vacío de estructuras de interpretación, $u=\left\{u_{1} \ldots u_{n}\right\} ; \mathbf{R}$ es una relación diádica entre los miembros de $\mathbf{S} \mathbf{y}$ $\mathrm{V}$ es una función que vincula interpretaciones con valores de verdad, entonces tenemos - en términos de las definiciones 1-5-que:

$$
\mathcal{M}, u_{1} \models \square \varphi \quad \text { sii para todo } u_{2} \mathrm{R} u_{1} \text {, en } u_{2} \models \varphi
$$

¿Es esta concepción semántica de los postulados aplicable a las teorías científicas entendidas cómo las entiende Carnap en FLM? Para responder a esta interrogante es necesario enfocarse en la noción de verdad. Según Andreas:

Es habitual decir que una teoría es verdadera si sus axiomas son proposiciones verdaderas en el dominio de las aplicaciones intencionales. ${ }^{13}$ Sin embargo, esta explicación es solamente aplicable si los símbolos no lógicos que aparecen en los axiomas están ya interpretados. Si, por contraste, los axiomas son parte de la interpretación de los símbolos no lógicos, como es el caso de los postulados y de los $t$, entonces, la asignación de los valores de verdad de los axiomas de la teoría no implica ninguna aserción sobre la verdad fáctica de la teoría

Es, por ende, razonable evaluar una teoría cuyos axiomas son postulados en términos de consistencia y no en términos de verdad. A pesar de que no hay un criterio de verdad en sentido de verdad fáctica, parece viable de un modo indirecto, ya que los postulados deberán satisfacer el requerimiento de consistencia en el contexto del sistema completo de asignación de los valores de verdad. (2008, p. 325).

Visto de este modo, los postulados se requieren para extender el lenguaje de un modo consistente a través de la introducción de los $t t$. De todos modos, a diferencia de las definiciones, no se requiere que sean extensiones conservativas del lenguaje ( $c f r$. Andreas 2008, p. 332)

\section{Méritos y problemas de la solución de Andreas}

\subsection{Ventajas de la solución}

Probablemente el acierto más importante de Andreas (2008, 2010) fue darse cuenta de que la lógica modal es adecuada para brindar

${ }^{13}$ Andreas suscribe aquí la visión de teoría científica de la denominada concepción estructuralista de la ciencia, inaugurada por Sneed (1971) y Stegmüller (1973), y desarrollada posteriormente por Balzer, Moulines y Sneed (1987). 
un enfoque semántico de las oraciones teóricas. Como vimos, la interpretación de una oración teórica es el conjunto de estructuras interpretativas en que esas oraciones teóricas son verdaderas. Por otra parte, el tratamiento de los $t t$ se ve favorecido en el marco del enfoque modal, pues éste permite lidiar con entidades carentes de extensión; sabemos que los $t t$, de ser verdaderos, no pueden serlo en el sentido de que a "verdadero" le damos en una semántica de primer orden (ya que de concederles extensión caeremos en el círculo sneedeano). Así, los contextos extensionales parecen poco propicios para desarrollar una semántica que dé cuenta de ellos; no así los contextos intensionales y es en esto donde se encuentra la novedad. Es dable destacar la importancia de poder contar con una semántica para oraciones teóricas, que incluye los postulados y que evita el círculo sneediano además de preservar las ventajas de la propuesta de Ramsey. No es menor la ventaja de tratarlos en una de las lógicas que más avances y líneas de investigación ha presentado en los últimos cincuenta años, como es el caso de la lógica modal.

Por último, señalemos que una característica que otorga la interpretación modal - considerada de nuestra parte una ventaja representacional - es que propone, en oposición a las formalizaciones de primer orden, un rango o conjunto de interpretaciones de la teoría (llamadas de "referencias múltiples" en la nota anterior). Como Andreas mismo dice:

La posición desarrollada aquí está, consecuentemente basada en el rechazo de la hipótesis de unicidad, es decir, la suposición de que el estado del mundo, o un fragmento del mismo, puede ser representado por una y sólo una estructura. Esta hipótesis sigue siendo válida sólo en relación con el lenguaje observacional. Por el contrario, en el lenguaje $L\left(V_{o}, V_{t}\right)$, se considera que cada estructura que es una extensión de la interpretación pretendida del lenguaje observacional y satisface los postulados, representa el estado del mundo, o un fragmento de éste. ${ }^{14}$ (2008, p. 332)

La consideración de las oraciones-t como oraciones verdaderas para un conjunto dado de interpretaciones va del todo acorde con las nuevas perspectivas acerca de la lógica modal, donde el operador modal " $\square$ " es considerado un cuantificador local:

14 "Para interpretar las fórmulas modales se precisan contextos intensionales, de referencias múltiples, que pueden ser de naturaleza temporal o incluir estados o situaciones diversos" (Manzano 2004, p. 195). Esta referencia múltiple es la que aparece perfectamente compatible con la negación de una interpretación única. 
Los operadores modales son una especie de cuantificadores, pero unos especiales "locales", que se refieren solamente a objetos "accesibles" a partir de los disponibles. Visto de este modo, los lenguajes modales no son extensiones, son más bien fragmentos de los clásicos, con formas restringidas de cuantificación - y esta debilidad es al mismo tiempo una clara fortaleza-. (van Benthem et al. 2010, p. 4 y ss.)

En resumen, Andreas logra proponer una interpretación alternativa - no extensional - a la interpretación de los términos-t que evita la circularidad que Sneed señala con respecto a la solución de la CH; al mismo tiempo que reconsidera seriamente la competencia de la concepción lingüística de las teorías. Propone esto en el marco de una semántica modal, basada en la consideración filosófica (proveniente de Carnap) de que los términos-t adquieren significado a través de una interpretación indirecta obtenida mediante postulados. En líneas generales, consideramos estos resultados buenos, novedosos y propicios para comenzar a extender las semánticas modales a algunas áreas de los planteamientos epistemológicos estructuralistas.

\title{
5.2. Límites de la solución
}

A pesar de lo anterior, podemos señalar algunos problemas representacionales del modelo. El modelo de Andreas consigue describir el comportamiento semántico de las oraciones teóricas entre las cuales se encuentran los postulados. Es factible, pues, adjudicar valores de verdad a los postulados en tanto que oraciones teóricas, pero no podemos saber - en el modelo- cuando una expresión es, además de una oración teórica, un postulado. En palabras del propio Andreas:

\begin{abstract}
Esta notación está destinada principalmente a representar la aserción de aquellos enunciados teóricos que no son postulados. Además, también representa la asignación de valores de verdad a los enunciados que son postulados, provistos por las reglas de verdad dadas en la definición 2, que se adoptan para tales enunciados. (2010, p. 376; las cursivas son nuestras.)
\end{abstract}

Tenemos una semántica para las oraciones teóricas; el problema es que no todas las oraciones teóricas son postulados, aunque todos los postulados son oraciones teóricas. Así, podemos considerar los postulados como un subconjunto de las oraciones teóricas. Ahora bien, ¿es posible distinguir los postulados de las oraciones teóricas en el modelo de Andreas? La representación que nos da este modelo de los postulados es un subconjunto de las oraciones teóricas, que se 
designa como " $\Phi_{T C}$ ", pero, para decirlo en dos palabras, no tenemos nada en el modelo que distinga las oraciones teóricas de los postulados a partir de las características lógicas propias de estos últimos, que deben considerarse en relación directa con la noción de cálculo, al menos en el contexto de FLM. Allí, Carnap se propone entre sus objetivos principales "precisar con claridad el papel de la lógica y las matemáticas aplicadas a la ciencia empírica" (1939, p. 2); esto lo lleva a precisar las ideas de cálculo e interpretación de manera independiente y posteriormente su aplicación en las ciencias empíricas. Tal es la estructura del libro. Si bien es cierto que en el resto de su obra Carnap rara vez habla de axiomas y postulados, ${ }^{15}$ en vez de sólo hablar de postulados, si lo hace en FML — que es el trasfondo de nuestra discusión-. Si alguien - basado en las propuestas carnapianas de 1956-1958 - quiere identificar axiomas con T-postulado puede hacerlo; nosotros nos ceñiremos a la terminología de FML:

Los cálculos que se aplican en ciencia se componen de dos partes: un cálculo lógico básico más un cálculo específico que se añade sobre él. El cálculo parcial específico generalmente no contiene reglas adicionales de inferencia, sino solamente enunciados primitivos adicionales llamados axiomas. (Carnap 1939, p. 38; las cursivas son del original.)

En cuanto a su interpretación los axiomas exigen —en la mayoría de los casos - algo más que una interpretación puramente lógica; exigen lo que Carnap denomina interpretación descriptiva. Sin embargo, lo que nos interesa destacar aquí, es el lugar que ocupan los axiomas en el cálculo: a partir de ellos es que se derivan los demás enunciados de la teoría; no todo enunciado de la teoría es un axioma pero todo enunciado es derivado a partir de ellos. Esta característica sintáctica esencial para la explicación carnapiana de los postulados ${ }^{16}$ no puede representarse en el modelo de Andreas.

En pocas palabras: la caracterización de Andreas — como él mismo declara en el Abstract - da un giro semántico a una caracterización que Carnap mantuvo como sintáctica en FLM. El resultado es un giro semántico donde, de lo sintáctico, ha quedado muy poco. Distinguir

\footnotetext{
${ }^{15}$ Incluso en FML, Carnap los maneja a veces como sinónimos. Cfr., p. 20 de ese texto.

${ }^{16}$ La explicación de Carnap del estatus lógico de los postulados está vinculada principalmente a su función sintáctica: los postulados son, de acuerdo con Carnap, axiomas no lógicos que pueden considerarse válidos en el cálculo y, por lo tanto, se pueden utilizar en cualquier derivación, como sucede con las definiciones (Andreas 2008, p. 321).
} 
los axiomas en el modelo es una de las cosas que se han sacrificado en el intento. Nosotros proponemos recuperar lo sintáctico del enfoque carnapiano de los postulados, sin por esto perder las ventajas proporcionadas por el giro semántico que Andreas propone.

La propuesta se compone de una caracterización de los axiomas, que acuerde con la propuesta sintáctica de Carnap en FLM, con ella, se extiende el modelo de Andreas a fin de obtener una representación de los axiomas "dentro" del modelo sin perder la perspectiva semántica modal.

\section{Nuestro modelo}

Para dar cuenta de la parte inferencial de modelos que describan la semántica de las oraciones teóricas, Andreas propone sistemas conocidos en la tradición modal con el nombre de S5. Éstos son - desde la perspectiva modal- sistemas modales normales, esto es, sistemas donde la regla modal N (Necesitación) y la regla proposicional MP (Modus Ponens) son válidos. S5 se caracteriza axiomáticamente por los axiomas $\mathrm{K}$, T, y 5 , que se validan en un marco determinado por una relación $\mathrm{R}$ de equivalencia. La elección de una relación $\mathrm{R}$ de equivalencia se funda en que, para caracterizar las oraciones con $t t$ como aquellas verdaderas en todas y cada una de las interpretaciones admitidas, es necesario que para la verdad de $\square \varphi$ (denotada por " $\models \square \varphi$ "), $\varphi$ sea verdadera en todos los estados del modelo que son considerados interpretaciones admisibles. Esto es, que $\varphi$ sea verdadera en todos de los estados $u \in S$, independientemente de desde donde éstos sean considerados; como una relación de equivalencia hace accesible a todos los estados desde cualquiera de ellos, la condición se cumple. Preservaremos, pues, el sistema S5 para nuestro modelo.

Ahora modificamos la Definición 2 a fin de agregar los operadores modales que nos hacen falta.

Definición 2': El lenguaje modal que utilizaremos (una ampliación del lenguaje modal básico) se define utilizando un conjunto $\Phi$ de letras proposicionales cuyos elementos son usualmente denotados por $p, q, r$, más dos operadores unarios: " $\mathcal{T}$ " y $\mathcal{A}$. Las fórmulas bien formadas $\varphi$ del lenguaje son dadas por la regla

$$
\varphi::=p|\perp| \neg \varphi|\varphi \vee \psi| \mathcal{A} \phi \mid \mathcal{T} \varphi
$$

Donde el rango de $p$ se establece sobre los elementos de $\Phi$.

Informalmente, la fórmula " $\mathcal{A} \varphi$ " se lee como " $\varphi$ es un axioma de la teoría $\mathrm{T}$ " у "T $\varphi$ " como " $\phi$ es una oración con $t t$ de la teoría $\mathrm{T}$ ". 
Con estos elementos podemos representar el conjunto de las fórmulas que interesa a nuestros fines.

Queremos recuperar la caracterización sintáctica de Carnap sin perder los logros semánticos de la de Andreas. Nuestra estrategia será entonces postular un enfoque mixto, esto es, un enfoque con elementos tanto sintácticos como semánticos.

En líneas generales, enriqueceremos un modelo como el que se definió anteriormente (Definición 4) a fin de representar los axiomas de la teoría, i.e., su costado sintáctico. Lo haremos adaptando una función conocida como función de awareness, utilizada en el ámbito de las lógicas epistémicas para atacar el problema de la omnisciencia lógica de los agentes. ${ }^{17}$ Nosotros la interpretaremos sin alusión alguna al conocimiento por lo que la denominaremos - conforme a nuestros intereses - función axiomática (pues nos permitirá representar los axiomas de la teoría modelada). Como dijimos antes, el enfoque que proponemos tiene tanto componentes sintácticos como semánticos. La intuición fundante es que los postulados son quienes restringen la interpretación de las estructuras. Es a partir de los postulados que las oraciones teóricas toman su significado. La representación de las fórmulas que componen $\Phi_{T C}$ es lo que garantiza una buena representación, pues la presencia de ellas, además de la interpretación del lenguaje observacional, es lo que permite establecer las oracionest sobre el conjunto de estructuras admisibles. ${ }^{18}$

Ahora sabemos que los postulados pueden explicarse poniendo énfasis en sus componentes semánticos (como Andreas) o en sus componentes sintácticos (como Carnap). Las características sintácticas de los postulados (las que derivan de su naturaleza lógica). Estarán representadas en el lenguaje por el operador $\mathcal{A}$, nuestro operador sintáctico. Sus características semánticas — como lo propone Andreas- estarán representados por el operador T.

Los marcos que utilizaremos siguen estando bien representados por la Definición 3 de la sección 4.2. Extenderemos el modelo modificando la Definición 4; denominamos a este tipo de modelos estructura axiomática.

${ }^{17}$ Para una muy buena explicación de la utilización, límites y posibilidades de este enfoque, véase Fagin, Halpern, Moses y Vardi (2003), capítulo 9, y especialmente la sección 5 .

${ }^{18}$ La gama de estructuras admisibles no sólo está determinada por la interpretación del lenguaje observacional prevista, sino también por los postulados $\Phi_{T C}$. Por lo tanto, el significado de una oración teórica está, en cierta medida, determinado por los axiomas de una teoría científica (Andreas 2010, p. 376). 
Definición $4^{\prime}$ : una estructura axiomática para el lenguaje modal básico es un tuplo $\mathcal{E} \mathcal{A}=(\mathcal{F}, \mathrm{V}, \mathrm{A})$, donde $\mathcal{F}$ es un marco y $\mathrm{V}$ es una función asignando a cada letra proposicional $p$ un subconjunto $\mathrm{V}(p)$ de $\mathrm{W}$ y $\mathrm{A}$ es una función asociando un conjunto de fórmulas con cada estado. Intuitivamente $\mathrm{A}(\mathrm{w})$ es el conjunto de fórmulas que son seleccionadas — con base en criterios sintácticos - para ser válidas en la interpretación w; en el sentido que nos interesa son fórmulas arbitrarias. Las distingue su lugar en el cálculo.

Ahora establecemos la semántica de nuestro modelo en base a modificaciones en la Definición 5.

Definición $5^{\prime}$ : suponemos que $w^{19}$ es un estado en una $\mathcal{E} \mathcal{A}=$ $(\mathcal{F}, \mathrm{V}, \mathrm{A})$; entonces definimos inductivamente la noción de fórmula satisfecha (o verdadera) en un estado $w$ como:

(i) $\mathcal{M}, w \models p$

(ii) $\mathcal{M}, w \models \perp$

(iii) $\mathcal{M}, w \models \varphi \wedge \psi$

(iv) $\mathcal{M}, w \models \neg \varphi$

(v) $\mathcal{M}, w \models \mathcal{T} \varphi$

(vi) $\mathcal{M}, w \models \mathcal{A} \varphi$

(vii) $\mathcal{M}, w \models \mathcal{P} \mathcal{T} \varphi$ sii $w \in \mathrm{V}(p)$ donde $p \in \varphi$

sii nunca es el caso

sii $\mathcal{M}, w \models \varphi$ y $\mathcal{M}, w \models \psi$

sii no se da que $\mathcal{M}, w \models \varphi$

sii para todo $\nu \in \mathbb{W}$ tal que $\nu \mathbf{R} w$ en $\mathcal{M}, \nu \models \varphi$

sii $\varphi \in \mathrm{A}(w)$

sii $\mathcal{M}, \mathrm{w}=\mathcal{A} \varphi$ y $\mathcal{M}, \mathrm{w}=\mathcal{T} \varphi$

La clásusula (v) es la que Andreas propone para otorgar una semántica a las oraciones teóricas; hemos agregado el operador modal unario $\mathcal{T}$, que nos dice que la fórmula que antecede, es una oración teórica.

La cláusula (vi) nos dice que si $\varphi$ pertenece al conjunto de fórmulas determinado por la función A para $w$, puede ser considerada una fórmula sintácticamente seleccionada para ser válida en $w$. El operador modal unario $\mathcal{A}$ nos dice de la fórmula que antecede, que ocupa el lugar de un axioma.

Ahora bien, como dijo Carnap anteriormente, los axiomas pueden ser axiomas lógicos, pertenecientes a lo que denomina cálculo básico, o axiomas del cálculo específico. Debemos entonces poder diferenciarlos, esto es, lo que hace la cláusula (vii) es proponer que para

${ }^{19}$ Como el lector habrá notado, $w$ en la notación modal canónica y u en la notación de Andreas, refieren, en el contexto de este trabajo, a lo mismo: la interpretación admisible de una teoría. Para no confundir, optamos por preservar $w$. La diferencia es sólo en la notación. En la sección 4.2. lo mostramos con la notación que Andreas utiliza. 
ser un axioma del cálculo específico (o un T-postulado) se requiere no sólo ocupar el lugar de un axioma en el cálculo, sino también poseer términos teóricos (característica que puede reflejarse gracias a la propuesta semántica de Andreas). Lo que tenemos entonces es que la conjunción de ambas características es lo que satisface al operador $\mathcal{P} \mathcal{T}$. Una oración $\varphi$ es un axioma de una teoría $\mathrm{T}$, sii es un axioma (sintácticamente hablando) y es una oración teórica (desde la perspectiva semántica).

Ahora podemos distinguir "desde dentro del modelo" las características sintácticas y semánticas de los axiomas de una teoría científica, es decir, "los enunciados específicos primitivos del cálculo [físico] en cuestión" (Carnap 1939, p. 57).

Una vez introducidos estos cambios, que modifican la capacidad de representación del modelo, mencionaremos algunas de las características que consideramos interesantes o destacables. Por motivos de espacio y por el estado actual de nuestros estudios, sólo señalaremos algunas de ellas.

Debido al costado sintáctico que incorporamos en el modelo podemos distinguir - por sus características lógicas - una oración como

$$
\text { (1) } \mathcal{M}, w \models \mathcal{P} \mathcal{T}(\varphi \rightarrow \psi) \rightarrow(\mathcal{P} \mathcal{T} \varphi \rightarrow \mathcal{P} \mathcal{T} \psi)
$$

de una oración como

(2) $\mathcal{M}, w \models \mathcal{T}(\varphi \rightarrow \psi) \rightarrow(\mathcal{T} \psi \rightarrow \mathcal{T} \psi)^{20}$

La oración (1), que puede leerse como: "las consecuencias de un axioma son siempre axiomas", en virtud del carácter esencialmente sintáctico de la función de axiomática $A$, no es válida en nuestro modelo. Esto revela que nuestro modelo recoge algunas intuiciones importantes: por ejemplo, que la consecuencia de un axioma, no tiene porque ser otro axioma. Sabemos que esto realmente funciona así: elegido un número de axiomas mínimo para una teoría $\mathrm{T}$, no quisiéramos que lo que de ellos se sigue se confunda con los axiomas mismos.

Por otro lado, la oración (2) sí es válida en nuestro modelo y dice que "la consecuencia de una oración teórica es siempre una oración teórica". Este enunciado está en conformidad con la distinción entre lenguajes teóricos y observacionales (sucede así porque el operador

${ }^{20}$ Ambas oraciones tienen la forma lógica del axioma $\mathrm{K}$, de las lógicas modales. Es una oración que es válida en todos los modelos modales normales. En su representación clásica: $\square(\varphi \rightarrow \psi) \rightarrow(\square \varphi \rightarrow \square \psi)$. 
$\mathcal{T}$ —que señala los $t t$ - está, por definición, cerrado bajo implicación material).

Por último, señalemos que estos modelos son aptos para ser considerados dinámicamente, perspectiva de interés si se pretende dar cuenta de aspectos históricos o pragmáticos ( $c f r$. van Benthem y Velázquez-Quesada 2010).

\section{Consideraciones finales}

En consonancia con Andreas, sostenemos que, mientras existe una única determinación de la extensión de un término definido mediante una definición, en la interpretación indirecta de un término por postulados obtenemos meramente una constricción impuesta sobre los valores admisibles de los $t t$, que no implica ésta una única determinación de los términos. Los significados de los $t t$ pueden estar conformados por varios postulados, hecho que refleja que comúnmente hay varios métodos de uso para uno y el mismo concepto científico. La interpretación para los $t t$ sería, entonces, holística. ${ }^{21}$

La divergencia filosófica más interesante de esta perspectiva con la semántica estándar consiste en rechazar la hipótesis de una única interpretación prevista de una teoría científica. Una vez que estamos convencidos de que hay $t t$, es decir, símbolos no lógicos que obtienen un significado determinado a través de los postulados de una teoría científica, el requerimiento de una única interpretación - esencial a la semántica estándar - ya no tiene sustento. ${ }^{22}$ Al igual que Andreas, sostenemos que, mientras existe una única determinación de la extensión de un término definido mediante una definición, en la interpretación indirecta de un término por postulados obtenemos meramente una constricción impuesta sobre los valores admisibles de los $t t$, que no implica ésta una única determinación de los términos. Los significados de los $t t$ pueden estar conformados por varios postulados, hecho que refleja que comúnmente hay varios métodos de uso para uno y el mismo concepto científico. La interpretación para los $t t$ sería, entonces, holística. En este sentido la semántica de mundos posibles

${ }^{21}$ Esta perspectiva de la interpretación, que reactualiza los aportes de la obra temprana de Carnap a una concepción holística del significado, puede ponerse en consonancia con obras posteriores de la CH, véase Hempel 1973, en las que se alude también al carácter global de la interpretación que se aplica a un sistema teórico como un todo.

${ }^{22}$ Cabe aclarar que en obras posteriores de Carnap (1956, 1966, 1975) así como en las versiones de Hempel (1966, 1970, 1973) hallamos concepciones que intentan salvar el problema en la versión estándar de la $\mathrm{CH}$ con respecto a los términos teóricos: la interpretación reductiva de éstos a términos de observación. 
parece la naturalmente adecuada para dar cuenta de las interpretaciones holísticas, debido a su carácter multirreferencial. Además de esto, se trata de un área muy estudiada y que está en constante expansión, lo que sugiere considerarla como un ámbito propicio para modelar distintas características de las teorías científicas.

Así, en términos generales, suponemos el ámbito de la semántica de mundos posibles como el ámbito adecuado donde reinterpretar ideas como las que rescatan las relecturas de Carnap, que resulta imposible desarrollar en el ámbito de la lógica de primer orden y de la concepción lingüística de las teorías.

En otras palabras: estas semánticas son el ámbito ideal para caracterizar formalmente el componente semántico de las concepciones que suponen las teorías científicas más como conjuntos de modelos que como conjuntos de enunciados. Nuestra reformulación de la propuesta de Andreas en términos de modelos de Kripke y su extensión a fin de hacer patente la distinción entre axiomas, postulados y $t t$ es una muestra de ello.

Por último, podemos señalar algunas posibles perspectivas en relación con las potencialidades del enfoque que asumimos, por ejemplo, los componentes pragmáticos que cada vez más buscan incorporarse a la caracterización de las teorías científicas — como su dinámicaintuitivamente no tendrían mejor lugar que una lógica de carácter intensional y dinámica (como la acuñada en van Ditmarsch et al. 2008), o los modelos alternativos al tratamiento clásico del operador "K" (Artemov 2004; Fitting 2005). Todas ellas son lógicas epistémicas que se dedican a tratar grupos de agentes más que a agentes individuales y parecen resultar ser un marco propicio para un tratamiento fecundo de problemas clásicos de la epistemología con vistas a futuros trabajos. ${ }^{23}$

\section{BIBLIOGRAFÍA}

Acero, J.J., 1994, "Sintaxis lógica, fisicalismo y la unidad de la ciencia", Revista de Filosofía, Universidad Complutense de Madrid, vol. 7, no. 12, pp. 421-433.

Andreas, H., 2013, "Theoretical Terms in Science", The Stanford Encyclopedia of Philosophy (Summer Edition), en Edward N. Zalta (ed.),

${ }^{23}$ Queremos agradecer especialmente a los árbitros anónimos que se ocuparon de la lectura crítica de este trabajo. Sin sus acertadas y agudas sugerencias y observaciones la calidad del material sería en mucho inferior. También agradecemos a aquellos que lo escucharon, criticaron y leyeron en versiones previas. 
[<http://plato.stanford.edu/archives/sum2013/entries/theoretical-terms-science/>. Fecha de consulta: 20/03/2015.]

Andreas, H., 2010, "A Modal View of The Semantics of Theoretical Sentences", Synthese, vol. 174, no. 3, pp. 367-383.

- 2008, "Another Solution to The Problem of Theoretical Terms", Erkenntnis, vol. 69, no. 69, pp. 315-333.

- 2007, Carnaps Wissenschaftslogik, Mentis, Paderborn.

Awodey, S., 2007, “Carnap's Quest for Analyticity: The Studies in Semantics", The Cambridge Companion to Carnap, en Michael Friedman y Richard Creath (eds.), Cambridge University Press, Cambridge, pp. 226247.

, 2004, "Evidence-Based Common Knowledge", Technical Report TR-2004018, Cuny Ph. D. Program in Computer Science.

Balzer, W., C. Moulines, J. Sneed, 1987, An Architectonic for Science, Reidel, Dordrecht.

Barwise, J y J. Etchemendy, 1999, Language, Proof and Logic, Leland Stanford Junior University, Center for the Study of Language and Information.

Blackburn, P., M. de Rijke, Y. Venema, 2001, Modal Logic, Cambridge University Press, Cambridge.

Carnap, R., 1988/1928, La construcción lógica del mundo, unAm, México.

—, 1985/1966, Fundamentación lógica de la física, Eds. OrbisHyspamérica, Buenos Aires.

- 1958, "Beobachtungssprache und theoretische Sprache", Dialectica, vol. 12, no. 3-4, pp. 236-248. [Versión en inglés utilizada: "Observational Language and Theoretical Language", en J. Hintikka (ed.), Rudolf Carnap. Logical Empiricist, Reidel Publishing Company, Dordrecht, pp. 75-86.]

_ 1956, "The Methodological Character of Theoretical Concept", en H. Feigel y M. Scriven (eds.), Minnesota Studies in the Philosophy of Science I., University of Minnesota Press, Minneapolis, pp. 38-76.

- 1952, "Meaning Postulates", Philosophical Studies, vol 3, no. 5, pp. 65-73.

, 1939, Foundations of Logic and Mathematics, University of Chicago Press, Chicago.

- 1937, The Logical Syntax of Language, Routledge, Londres.

Coffa, A., 1991, The Semantic Tradition Kant to Carnap: To the Vienna Station, Cambridge University Press, Cambridge.

Davidson, D., 1963, "The Method of Extension and Intension", en The Philosophy of Rudolf Carnap, P. Schilpp (ed.), Northwestern University La Salle, Illinois, Open Court, pp. 311-399.

Fagin, R., J. Halpern, Y. Moses, M. Vardi, 2003, Reasoning about Knowledge, MIT Press, Cambridge, Mass./Londres, Inglaterra.

Fitting, M., 2005, “A Logic of Explicit Knowledge", en L. Behounek y M. Bilkova (eds.), Logica Yearbook 2004. <http://comet.lehman.cuny.edu> 
Friedman, M., 2011, "Carnap on Theoretical Terms: Structuralism without Metaphysics", Synthese, vol. 180, no. 2, pp. 249-263.

—, 1999, Reconsidering Logical Positivism, Cambridge University Press, Cambridge.

Hempel, C., 1989/1973, “El significado de los términos teóricos: una crítica de la concepción empirista estándar", en L. Olivé y R. Pérez Ransanz (comps.), Filosofía de la ciencia: teoría y observación, Eds. S. XXI, México, pp. 439-453.

- 1987/1966, Filosofía de la ciencia natural, Alianza Editorial, Madrid, XI ed.

— 1986/1970, "Sobre la 'concepción estándar' de las teorías científicas", en J.L. Rolleri (comp.), Estructura y desarrollo de las teorías científicas, Universidad Nacional Autónoma de México-Instituto de Investigaciones Filosóficas, México, pp. 141-166.

Manzano, M., 2006, "Lógica modal", en Summa Logicae, <http://logicae. usal.es/>

Putnam, H., 1989/1962, “Lo que las teorías no son”, en L. Olivé y R. Pérez Ransanz (comps.), Filosofía de la ciencia: teoría y observación, Eds. S. XXI, México, pp. 312-329.

Sneed, J., 1971, The Logical Structure of Mathematical Physics, Reidel Publishing Company, Dordrecht.

Stegmüller, W., 1973, Probleme und Resultate der Wissenschaftstheorie und Analytischen Philosophie, vol. II, Theorie and Erfahrung, Subvolumen II: Theorienstrukturen und Theoriendynamik, Springer, Berlín.

Suppe, F., 1979/1974, La estructura de las teorías científicas, trad. Pilar Castrillo y Eloy Rada, Editora Nacional, Madrid.

van Benthem, J., F. Velázquez-Quesada, 2010, "The Dynamics of Awareness", Synthese, vol. 177, S.1, pp. 5-27.

van Ditmarsch, H. Kooi, W.V. Van der Hoek, 2008, Dynamic Epistemic Logic, Synthese Library, Springer, p. 337.

Recibido el 5 de agosto de 2014; revisado el 12 de junio de 2015; aceptado el 28 de agosto de 2015. 